\title{
Article \\ Effect of Vacancy Defects on the Vibration Frequency of Graphene Nanoribbons
}

\author{
Hong Guo $(\mathbb{1}$ and Jing Wang *(-) \\ Xinjiang Key Laboratory of Solid State Physics and Devices, College of Physics Science and Technology, \\ Xinjiang University, Urumqi 830046, China; gh530468@163.com \\ * Correspondence: wjxju@163.com
}

check for updates

Citation: Guo, H.; Wang, J. Effect of Vacancy Defects on the Vibration Frequency of Graphene Nanoribbons. Nanomaterials 2022, 12, 764. https:// doi.org/10.3390/nano12050764

Academic Editors: Gang Zhang, Kai Ren, Bin Ding and Henrich Frielinghaus

Received: 27 December 2021 Accepted: 22 February 2022 Published: 24 February 2022

Publisher's Note: MDPI stays neutral with regard to jurisdictional claims in published maps and institutional affiliations.

Copyright: () 2022 by the authors. Licensee MDPI, Basel, Switzerland. This article is an open access article distributed under the terms and conditions of the Creative Commons Attribution (CC BY) license (https:// creativecommons.org/licenses/by/ $4.0 /)$.

\begin{abstract}
Graphene is a type of two-dimensional material with special properties and complex mechanical behavior. In the process of growth or processing, graphene inevitably has various defects, which greatly influence the mechanical properties of graphene. In this paper, the mechanical properties of ideal monolayer graphene nanoribbons and monolayer graphene nanoribbons with vacancy defects were simulated using the molecular dynamics method. The effect of different defect concentrations and defect positions on the vibration frequency of nanoribbons was investigated, respectively. The results show that the vacancy defect decreases the vibration frequency of the graphene nanoribbon. The vacancy concentration and vacancy position have a certain effect on the vibration frequency of graphene nanoribbons. The vibration frequency not only decreases significantly with the increase of nanoribbon length but also with the increase of vacancy concentration. As the vacancy concentration is constant, the vacancy position has a certain effect on the vibration frequency of graphene nanoribbons. For nanoribbons with similar dispersed vacancy, the trend of vibration frequency variation is similar.
\end{abstract}

Keywords: graphene nanoribbons; vacancy defect; vibration frequency; molecular dynamics simulation

\section{Introduction}

Graphene has excellent elastic properties and high intrinsic strength [1-4], as well as extraordinary mechanical [5-7], electrical [8,9], and other physical [10-12] properties, making it one of the most promising materials in modern technology. Therefore, graphene is widely used in new energy research fields such as nanosensors [13-15], composite materials [16], drug delivery agents [17], solar cells, and supercapacitors [18,19], and also has an irreplaceable position in many fields, thus attracting much attention. It is clear that these properties depend on the structure of the hexagonal graphene lattice and the strong inplane $s p^{2}$ bonds between the carbon atoms. The mechanical property of graphene is crucial for its reliable application, both from a technical point of view and from the perspective of understanding its fundamental interest in physics deformation [20-22]. However, in the actual preparation of graphene, it is almost impossible to obtain perfectly structured graphene, and some defects such as Stone-Thrower-Wales (STW) defects [23], vacancy defects [24], grain boundaries [25], and adsorbed atoms [26] are inevitable. The most common defects are vacancy defects. The structural symmetry and integrity of graphene can be reduced due to the presence of lattice defects, which changes the property of graphene. Due to the presence of vacancy defects, the tensile strength of graphene is reduced [27]. Wang et al. [28] found that both Stone-Thrower-Wales and vacancy defects can lead to a significant reduction in the mechanical strength of graphene. Rahmandoust et al. [29] investigated dislocation, vacancy, and Stone-Wales defects in the semiconductor graphene quantum dot model, showing that mechanical property reduction is close to the experimentally measured values, while the increase in the number of layers does not significantly affect the final results. Park et al. [30] investigated the effect of defect size on the mechanical 
properties of graphene, in which large-sized vacancy defects have a higher tensile modulus and lower fracture strain compared to small-sized vacancy defects. Motamedi et al. [31] studied the effect of central cracking on graphene, and Young's modulus of graphene decreased from 905 GPa to 697 GPa as the crack length increased. Lopez-Polin et al. [32] conducted a systematic study on the elastic modulus and strength of graphene with controllable defect density; the results show that Young's modulus increases with increasing defect density, elastic modulus decreases with defect inclusions for higher density defects, and fracture strength decreases with increasing defect density. $\mathrm{Ng}$ et al. [33,34] used molecular dynamics (MD) simulations to study the change in thermal conductivity of graphene under two different chiralities and the relationship between chirality and STW defect density. The results showed that the thermal conductivity decreased with increasing STW defect density, independent of chirality. Lee et al. [35] measured the elastic properties and intrinsic fracture strength of freestanding monolayer graphene films using nanoindentation and showed that atomically perfect nanoscale materials could be tested for deformation using mechanical methods over a range well beyond the linear range. Therefore, the introduction of defects has an important effect on the structural properties of graphene. In this paper, we use molecular dynamics simulations to investigate the effects of different defect concentrations and defect locations on the vibration frequency of monolayer graphene.

\section{Physical Model and Simulation Method}

In this paper, the vibration frequencies of monolayer graphene nanoribbons with different defect concentrations and different defect locations were calculated using the Forcite Tools module of MS (Materials Studio 8.0) software and the molecular dynamics method. It is predictable that ideal graphene nanoribbons and graphene nanoribbons with different vacancy defects produce different vibrations under the same simulation conditions. The structure of graphene nanoribbons was fixed with the width $(3.935 \mathrm{~nm})$ and the thickness $(2.0 \mathrm{~nm})$, and the length increased from $8.379 \mathrm{~nm}$ to $23.718 \mathrm{~nm}$.

The types of vacancy defects in graphene we discuss in this paper (based on a supercell with the number of atoms $8 \times 8 \times 1$ ) are shown in Figure 1 , where the dark spheres represent carbon atoms and the light spheres represent missing atoms. $\mathrm{V}-i(i=1,2, \ldots \ldots, 6)$ indicates the type of vacancy and $i$ is the number of missing atoms in a supercell. For example, $3(2+1)$ indicates that the number of missing atoms in the supercell is three, and $(2+1)$ indicates the combination of missing atoms. The structure of graphene nanoribbons was obtained by expanding the supercells of the above model according to the requirement. Figure 2 a show a graphene supercell containing a vacancy type of $6(3+3)$. A silicon probe was placed directly on top of the graphene nanoribbon, which is close to the nanoribbon but does not bind to the atoms on the surface of the graphene. The structurally optimized nanoribbons bent due to the presence of Van der Waals forces between the silicon probe and the graphene nanoribbons [36,37]. Subsequently, the silicon probe was removed as the nanoribbon was bent to a certain extent, which is the initial bending state of the graphene nanoribbon shown in Figure $2 b$. Then, dynamic mechanical simulations of bent nanoribbons were performed in the NVE ensemble. Figure $2 \mathrm{c}, \mathrm{d}$ show the vibration of the graphene nanoribbon at a certain time, respectively. The COMPASS II force field [38] was selected for all structure optimization during the simulation, the simulation temperature was $300 \mathrm{~K}$ and the truncation radius was $15.5 \AA$. As the length of the graphene nanoribbon increased, the vibrational period of its energy also increased; therefore, the simulation time was set to $60 \mathrm{ps}$ with a time step of $1 \mathrm{fs}$ [39]. The total energy of the nanoribbon was obtained by simulation, as shown in Figure 3, and the kinetic energy and the potential energy fluctuated during the vibration of the nanoribbon. Kinetic energy and potential energy are converted into each other, and the vibration period can be obtained according to the curve of kinetic energy and potential energy, from which the vibration frequency of the graphene nanoribbons can also be obtained. 


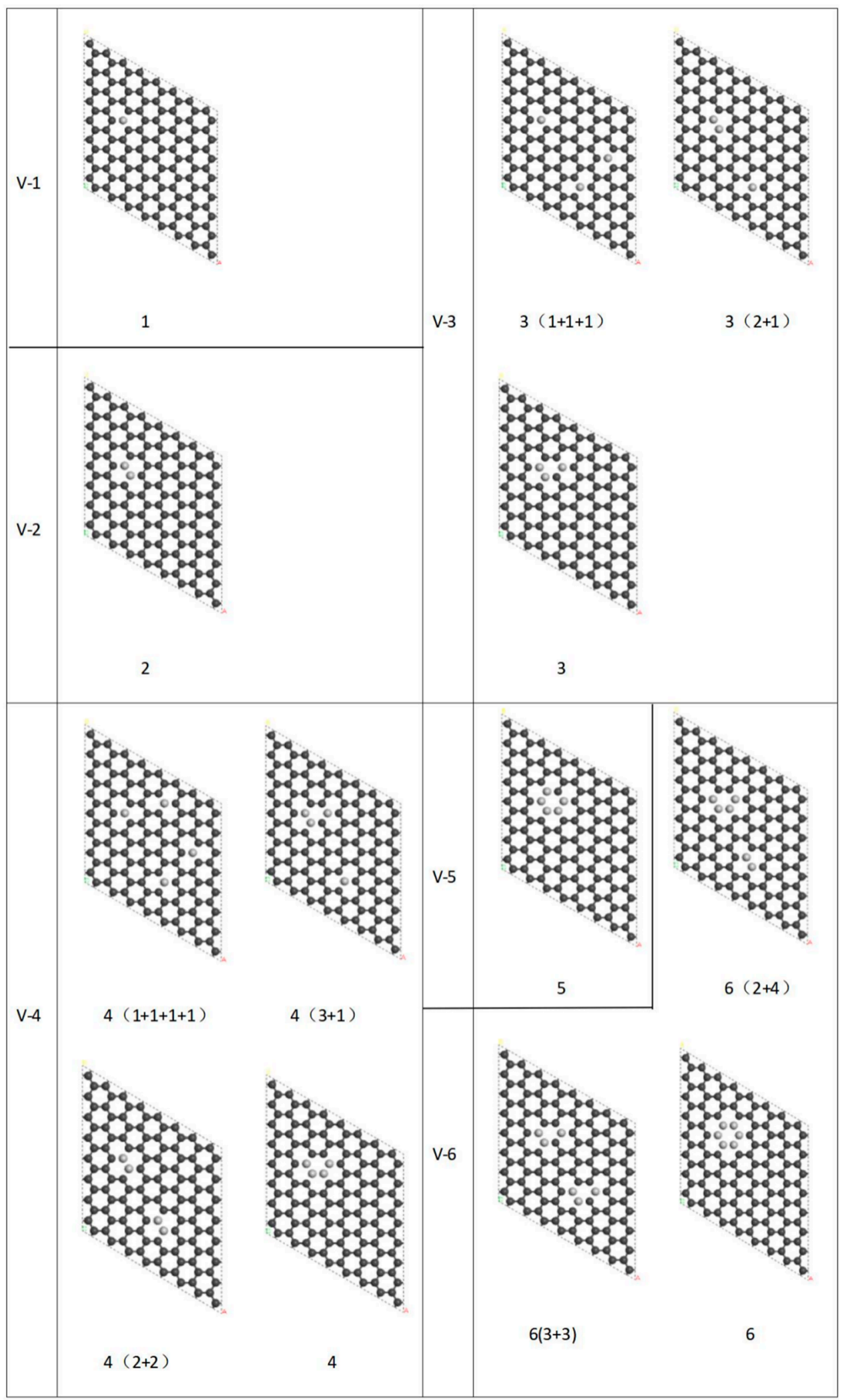

Figure 1. Types of vacancy defect in a supercell of graphene. The dark spheres represent carbon atoms and the light spheres represent missing carbon atoms. 
(a)

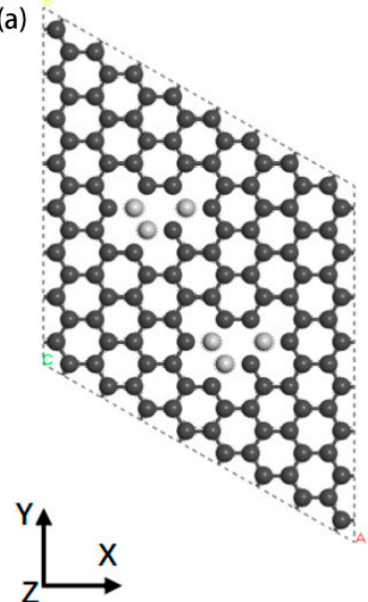

(b)
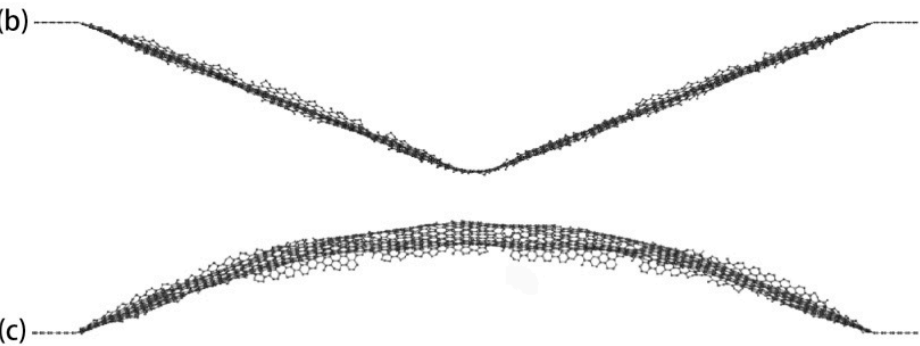

(d)

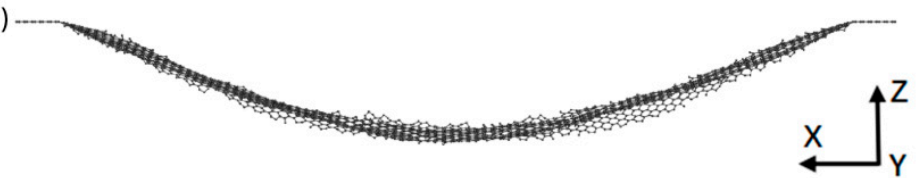

Figure 2. Graphene nanoribbons (a) Graphene supercell containing vacant $6(3+3)$ sites; (b) Initial bending state; $(\mathbf{c}, \mathbf{d})$ are vibration states at a certain time.

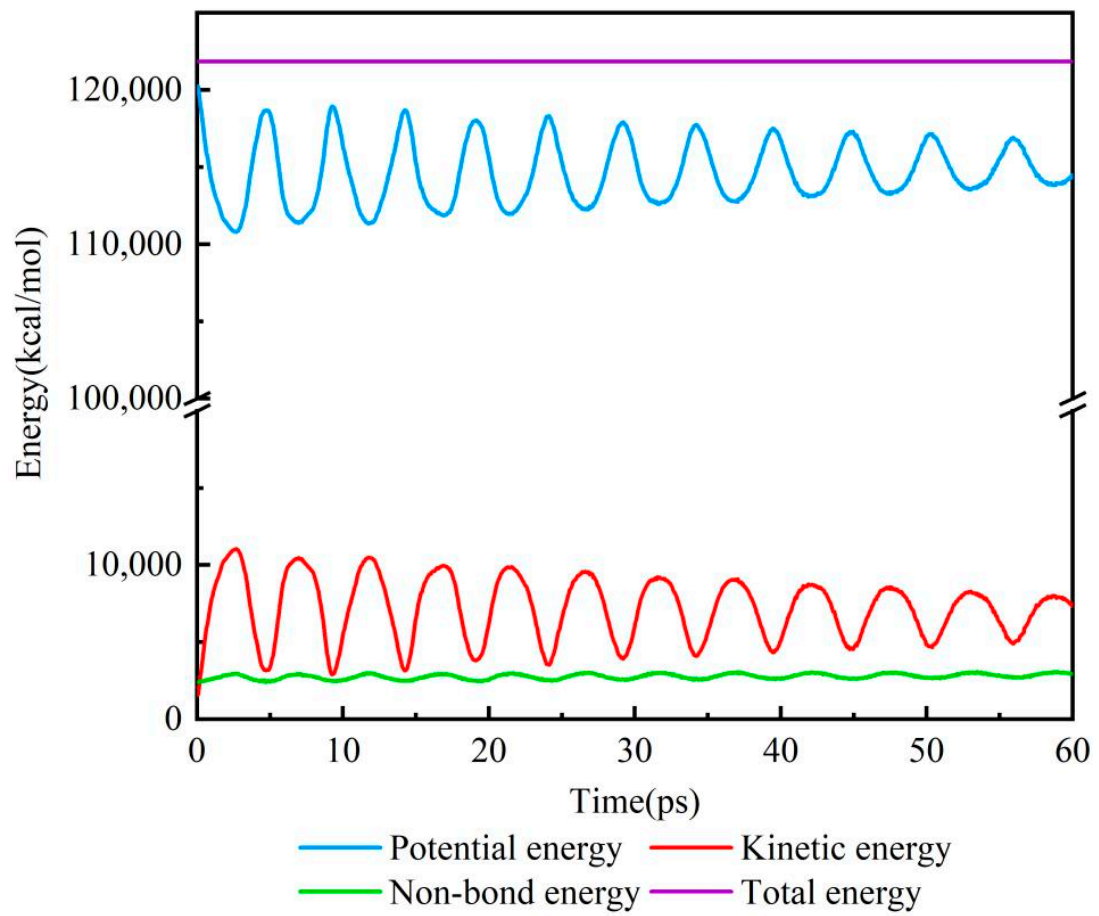

Figure 3. Energy of graphene nanoribbons during vibration.

\section{Results and Discussion}

The structure of different vacancy supercells was expanded to study the effect of the vacancy concentration of graphene nanoribbons with different lengths on their vibration frequencies. As the vacancy concentration was certain, the effect of the vacancy position of graphene nanoribbons on their vibration frequency was also explored.

The vibration frequency of graphene nanoribbons with different vacancy concentrations were obtained from the energy of the simulation results using molecular dynamics method, as shown in Figure 4. It can be seen that the vibration frequency of the vacancyfree graphene nanoribbon (Ideal) decreased monotonically as the length of the graphene nanoribbon increased. The existence of vacancy defects leads to significant changes in the structure of carbon atoms around the vacancies, as well as in the bond-to-bond strength and bonding mode. Taking the single-vacancy supercell (V-1) (shown in Figure 1), for example, the length of the nearest C-C bond of the ideal graphene nanoribbon was $1.403 \AA$, 
the distance of the second nearest neighbor $\mathrm{C}$ atom was $2.327 \AA$, the distance of the third nearest neighbor $\mathrm{C}$ atom was $2.764 \AA$, and the adjacent C-C-C bond angle was $122.984^{\circ}$. However, in the nanoribbon with vacancy, the length of the C-C bond was $1.483 \AA$, the distance of the second nearest neighbor $C$ atom was $2.430 \AA$, the distance of the third nearest neighbor $C$ atom was $3.015 \AA$, and the bond angle between adjacent atoms was $126.616^{\circ}$. This comparison means that the positions of carbon atoms in the nanoribbons changed. That is to say, the bond lengths and bond angles between $C$ atoms were significantly changed. Due to the existence of vacancy defects, the bond lengths and bond angles generally increased after structure optimization, which led to the structural change of the carbon atoms around the vacancies, thus affecting the structure of the nanoribbons.

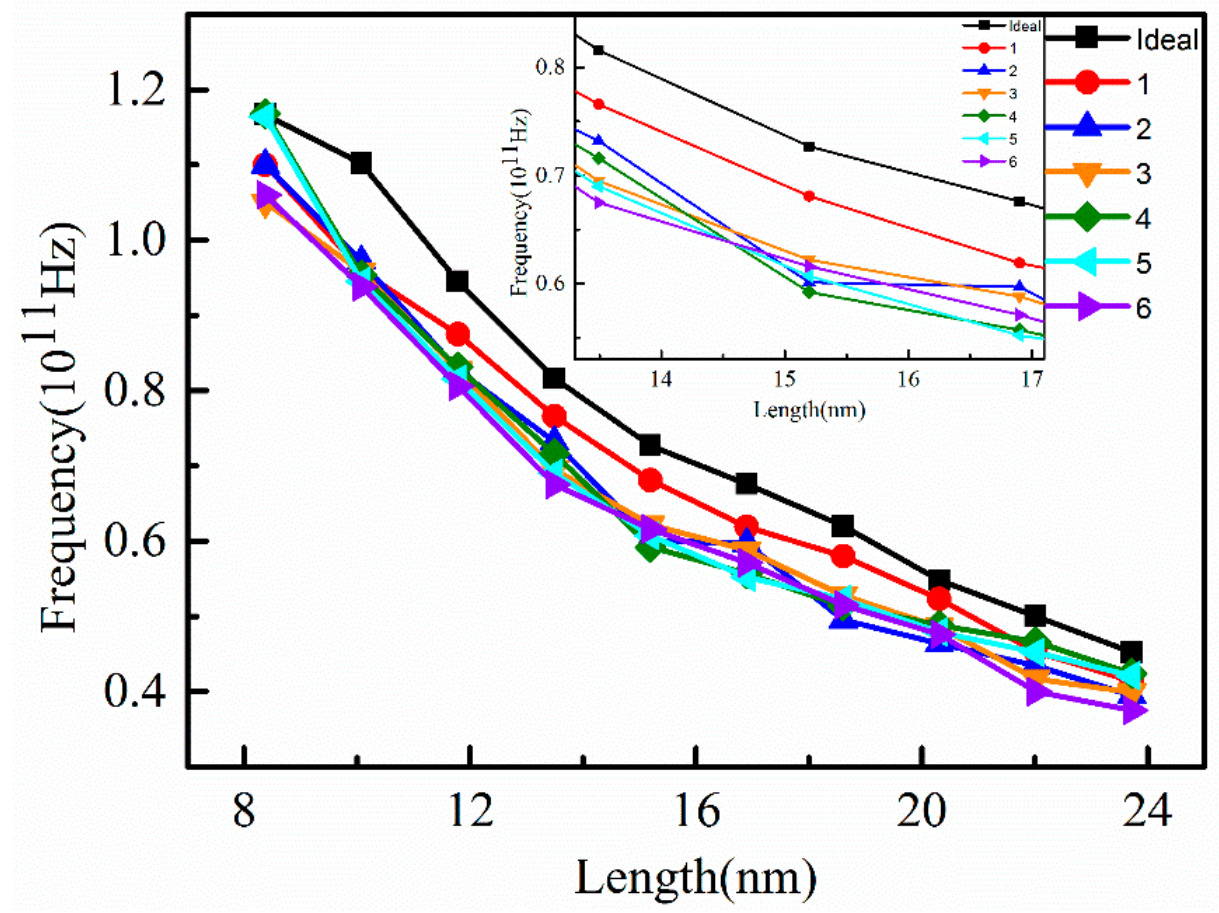

Figure 4. Vibration frequency with length of graphene nanoribbons with different vacancy concentrations.

Therefore, the variation of bond length and bond angle mades the $\mathrm{C}-\mathrm{C}$ bond energy of graphene vancancy-containing nanoribbons smaller than the ideal structure, which meant that the graphene nanoribbons containing vacancies acquired less deformation energy than that of the ideal graphene nanoribbons after bending. In addition, the deformation energy of graphene nanoribbons with multiple vacancies was converted to smaller vibration energy in the NVE system synthesis. Since the vibration energy was proportional to the square of the vibration frequency, the vibration frequency of graphene nanoribbons containing vacancies was less than that of ideal graphene nanoribbons.

In contrast, the inset shows that the vibration frequency of graphene nanoribbons containing different vacancy concentrations decreased with increasing length as the nanoribbon length increased from $13 \mathrm{~nm}$ to about $17 \mathrm{~nm}$. In particular, the vibration frequency of the graphene nanoribbons containing five vacancies decreased sharply; the reduction was greater than that of other vacancies. When the length exceeded $20 \mathrm{~nm}$, the reduction in the vibration frequency changed slowly. The vibration frequencies of the nanoribbons containing single vacancies were significantly higher than those of other vacancies, mainly because the interactions between atoms around the vacancies changed little. In addition, the bond lengths and bond angles between the carbon atoms in nanoribbons containing single vacancies varied less, which had less influence on the vibration frequency.

Except for a single vacancy, the decreasing trend of vibration frequency of other vacancies was not obvious. In general, compared with ideal graphene nanoribbons, the 
vibration frequency of nanoribbons containing vacancies decreased with increasing vacancy concentration. Therefore, vacancy concentration has a certain effect on the vibration frequency of nanoribbons.

In addition, dynamic simulation of graphene nanoribbons with different vacancy positions was carried out while the vacancy concentration was kept constant to further understand the influence of different vacancy positions on the vibration frequency of nanoribbons. As shown in Figure 5, it can be seen that the vibration frequency of the graphene nanoribbons without vacancy (ideal) and with a certain vacancy concentration but different vacancy positions (V-3) both decreased monotonically with the increase in length of the graphene nanoribbons. There was a significant difference in the vibration frequency between the ideal and the vacancy nanoribbon. The vibration frequency of graphene nanoribbons was not significantly affected by different vacancy positions at the same vacancy concentration. When the nanoribbon length was less than $10 \mathrm{~nm}$, the vibration frequencies of the dispersed vacancies $3(2+1)$ and $3(1+1+1)$ were greater than that of the concentrated vacancies 3. Especially in the length range of 10-20 nm, the vibration frequency of graphene nanoribbons with dispersed vacancy was generally smaller than that of the vacancy concentrated nanoribbons, indicating that the vacancy location had a certain influence on the vibration frequency of graphene nanoribbons. As the nanoribbon length became larger than $20 \mathrm{~nm}$, the influence became insignificant.

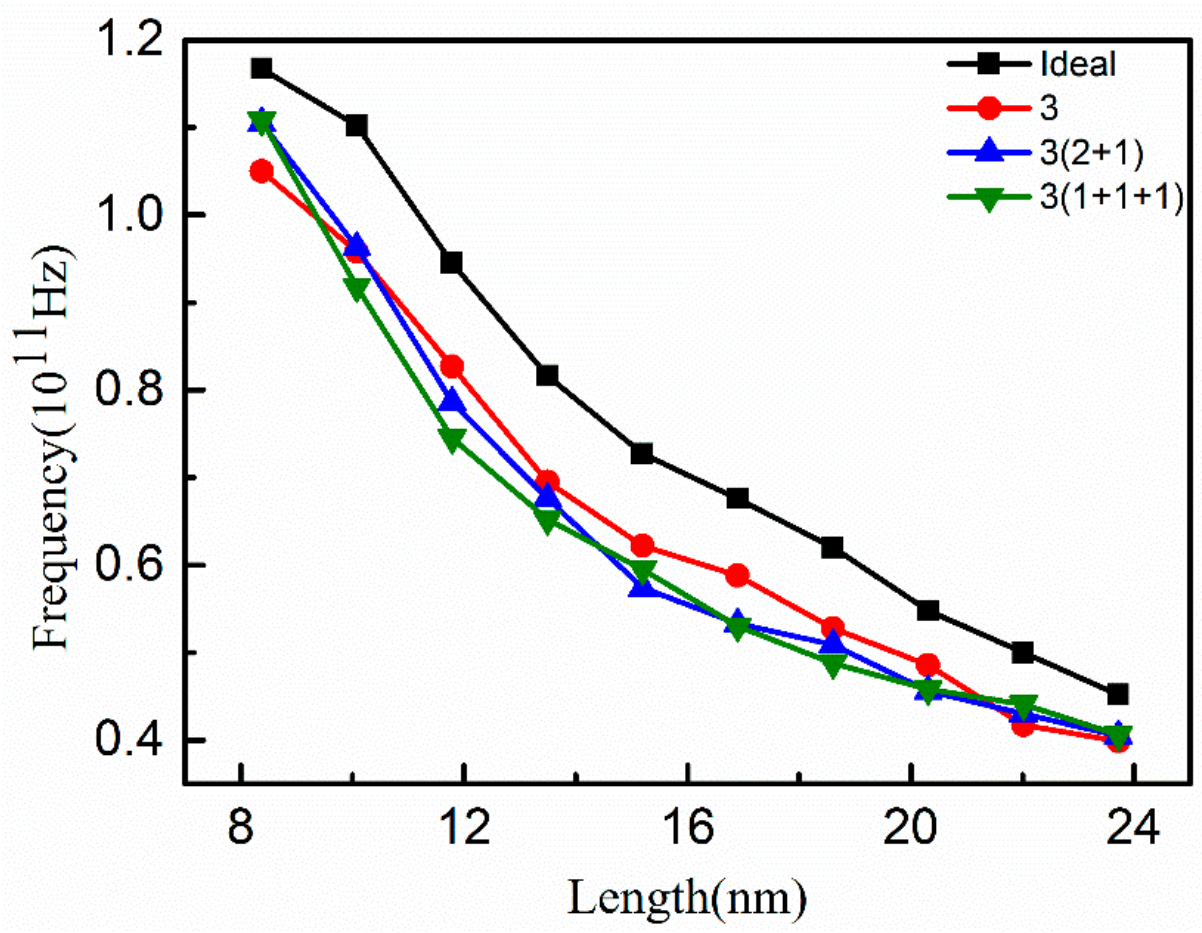

Figure 5. Variation of vibration frequency of graphene nanoribbons at different vacancy positions (V-3) with length.

As the number of the vacancy in a supercell was four $(\mathrm{V}-4)$, the effect of different vacancy positions on the vibration frequency of graphene nanoribbons with (V-4) were discussed, as shown in Figure 6. When the nanoribbon length was about $8.5 \mathrm{~nm}$, the vibration frequency of the graphene nanoribbons with concentrated vacancies was identical to that of the ideal graphene nanoribbon, indicating that the concentrated vacancies had less effect on the vibration frequencies of the nanoribbon with this length. When the length of the nanoribbon increased from $8.5 \mathrm{~nm}$ to about $13.5 \mathrm{~nm}$, the vibration frequency of nanoribbons with concentrated vacancies and dispersed vacancies decreased sharply, and the decrease of vibration frequency of dispersed vacancies was smaller than that of concentrated vacancies. As the nanoribbons were longer than $15 \mathrm{~nm}$, the decrease of the 
vibration frequency of nanoribbons with concentrated and dispersed vacancies decreased, and the vibration frequencies of nanoribbons with dispersed vacancies at different positions were almost the same, which indicated that the position of vacancies had little effect on the vibration frequency. By comparing Figures 5 and 6, it can be found that different vacancy positions have a certain influence on the vibration frequency of the nanoribbon. In general, the effect of concentrated vacancies is small, while that of dispersed vacancies is large. When the combination of the vacancy is similar, such as $4(2+2)$ and $4(1+3)$, the vibration frequencies of the nanoribbon are almost the same.

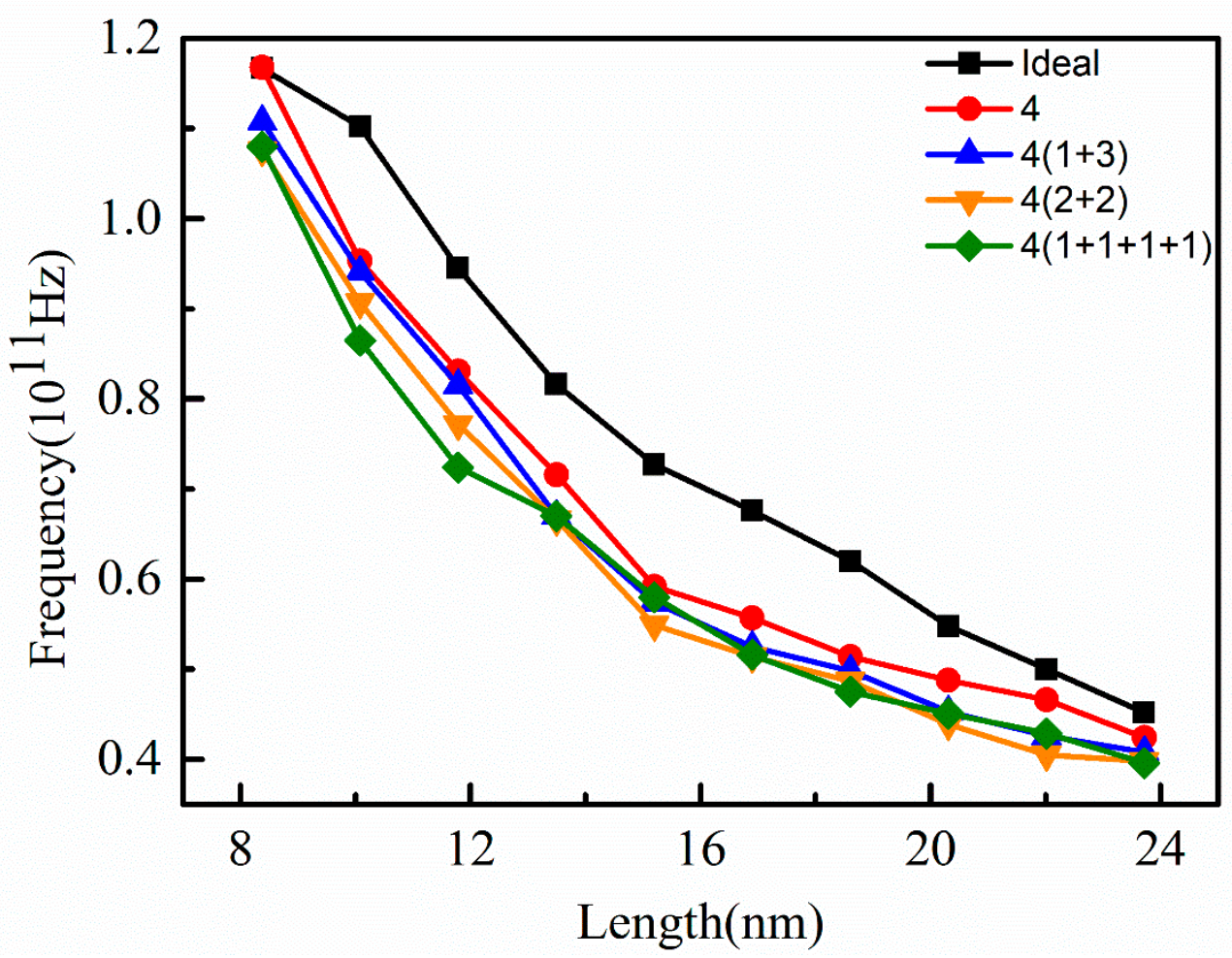

Figure 6. Variation of vibration frequency of graphene nanoribbon at different vacancy positions (V-4) with length.

Compared with other vacancies, the vibration frequency of graphene nanoribbons containing vacancies (V-6) is significantly smaller than that without vacancies (Ideal) when the nanoribbon length is small, as shown in Figure 7 . When the nanoribbon lengths were $8.5 \mathrm{~nm}$ and $13.5 \mathrm{~nm}$, the effect of concentrated vacancies and dispersed vacancies on the vibration frequency was not much different as the vacancy concentration was certain. When the length of the nanoribbon was between $13.5 \mathrm{~nm}$ and $20 \mathrm{~nm}$, the vibration frequency of the nanoribbon with dispersed vacancies was smaller than that of concentrated vacancies, which is mainly related to the bonding mode and strength of atoms around the vacancy. As the length was larger than $20 \mathrm{~nm}$, the vibration frequency was almost independent of the vacancy position with the increase of nanoribbon length. In general, as the combination of the vacancy was similar, the vibration frequencies of the nanoribbon were almost the same, such as $6(2+4)$ and $6(3+3)$, which coincided with that of $4(1+3)$ and $4(2+2)$, shown in Figure 6 . This means that the more similar the vacancy positions, the closer the vibration frequency of graphene nanoribbons. 


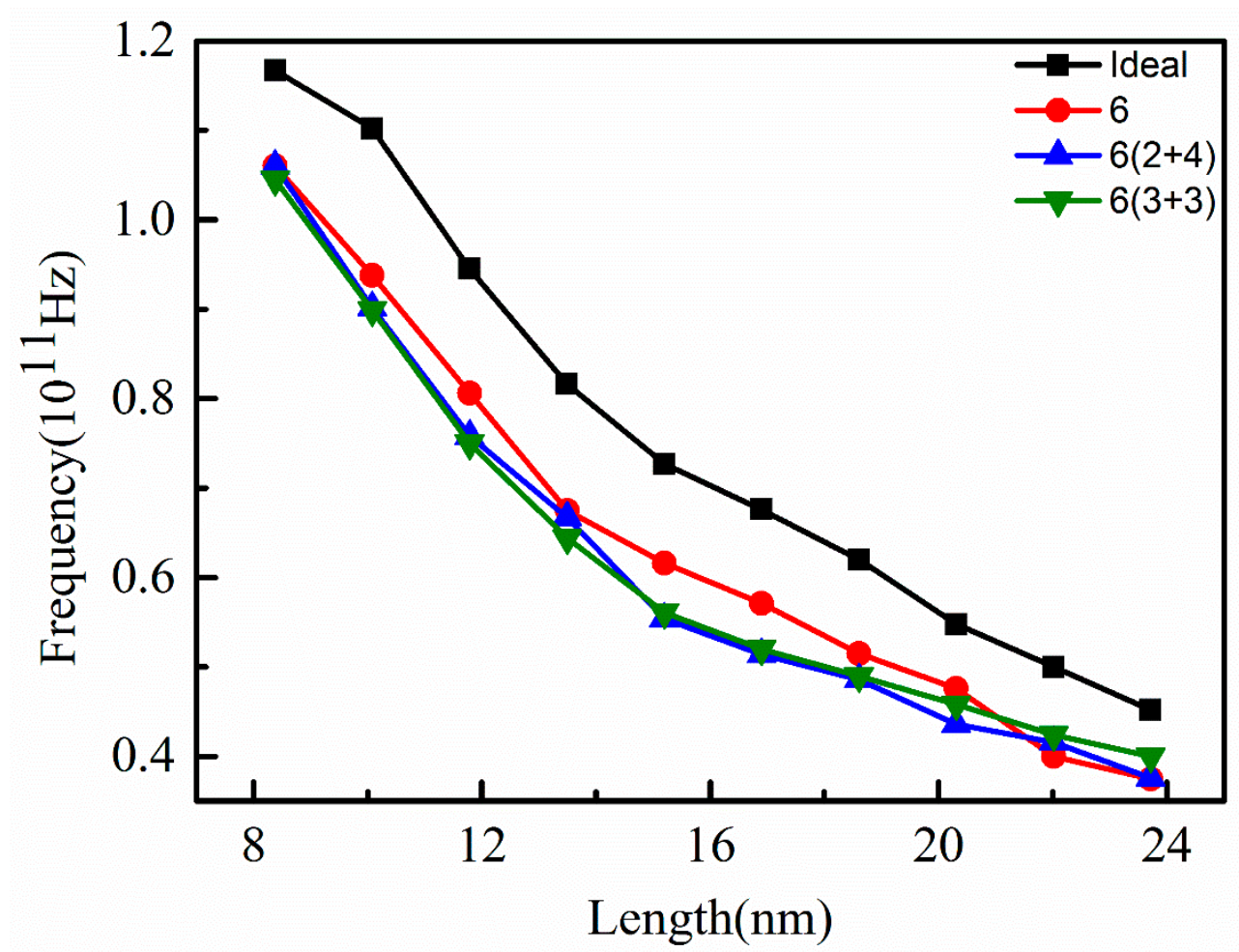

Figure 7. Variation of vibration frequency of graphene nanoribbon at different vacancy positions (V-6) with length.

\section{Conclusions}

Defects are inevitable in the actual preparation and processing of graphene. The effects of vacancy defect concentrations and vacancy positions on the vibration frequency of monolayer graphene were studied using molecular dynamics simulations. The results show that vacancy defects reduce the vibration frequency of the graphene nanoribbon, and both vacancy concentration and vacancy position have an effect on the vibration frequency of graphene nanoribbons. Regardless of the presence of vacancy defects, the vibration frequency of graphene nanoribbons decreases with the increase of length. Compared with ideal nanoribbons, nanoribbons with vacancies have smaller vibration frequencies. The vibration frequency of nanoribbons decreases with increasing vacancy concentration, especially when the length of the nanoribbon is small.

Furthermore, the vibration frequency of graphene nanoribbons with different vacancy positions showed little difference. The vibration frequency of the nanoribbon with dispersed vacancy was less than that of the nanoribbon with concentrated vacancy. As the vacancy positions were similar, the vibration frequency of graphene nanoribbons was close. In conclusion, both vacancy concentration and vacancy position have a certain effect on the vibration frequency of graphene nanoribbons, but the size effect of nanoribbons is the main factor.

Author Contributions: Conceptualization, J.W.; methodology, H.G.; software, H.G.; validation, J.W.; formal analysis, H.G.; investigation, H.G.; resources, J.W.; data curation, H.G.; writing-original draft preparation, H.G.; writing-review and editing, J.W.; visualization, H.G.; supervision, J.W.; project administration, J.W.; funding acquisition, J.W. All authors have read and agreed to the published version of the manuscript.

Funding: This study was supported by the National Natural Science Foundation of China (grant number 22066024).

Institutional Review Board Statement: Not applicable.

Informed Consent Statement: Not applicable. 
Data Availability Statement: The data presented in this study are available on request from the corresponding author.

Acknowledgments: Thanks to Xinjiang Key Laboratory of Solid State Physics and Devices and College of Physical Science and Technology of Xinjiang University for their support.

Conflicts of Interest: The authors declare no conflict of interest.

\section{References}

1. Bhadra, S.; Khastgir, D.; Singha, N.K.; Hee-Lee, J. Progress in polymer science. Polym. Int. 2010, 35, $1275-1350$.

2. Das, T.K.; Prusty, S. Graphene-based polymer composites and their applications. Polym. -Plast. Technol. Eng. 2013, 52, 319-331. [CrossRef]

3. Jing, N.; Xue, Q.; Ling, C.; Shan, M.; Zhang, T.; Zhou, X.; Jiao, Z. Effect of defects on Young's modulus of graphene sheets: A molecular dynamics simulation. Rsc Adv. 2012, 2, 9124-9129. [CrossRef]

4. Verdejo, R.; Bernal, M.M.; Romasanta, L.J.; Lopez-Manchado, M.A. Graphene filled polymer nanocomposites. J. Mater. Chem. 2011, 21, 3301-3310. [CrossRef]

5. Xiao, J.R.; Staniszewski, J.W., Jr. Tensile behaviors of graphene sheets and carbon nanotubes with multiple Stone-Wales defects. Mater. Sci. Eng. A 2010, 527, 715-723. [CrossRef]

6. Pei, Q.X.; Zhang, Y.W.; Shenoy, V.B. A molecular dynamics study of the mechanical properties of hydrogen functionalized graphene. Carbon 2010, 48, 898-904. [CrossRef]

7. Zhao, X.; Zhang, Q.; Chen, D.; Lu, P. Enhanced mechanical properties of graphene-based poly(vinyl alcohol) composites. Macromolecules 2010, 43, 2357-2363. [CrossRef]

8. Lee, E.; Zhi, L.; Burghard, M.; Muellen, K.; Kern, K. Electrical properties and photoconductivity of stacked-graphene carbon nanotubes. Adv. Mater. 2010, 22, 1854-1857. [CrossRef]

9. Park, S.; Ji, W.S.; An, J.; Oh, J.; Lee, S.; Lee, W.; Potts, J.R.; Byun, J.H.; Ruoff, R.S. The effect of concentration of graphene nanoplatelets on mechanical and electrical properties of reduced graphene oxide papers. Carbon 2012, 50, 4573-4578. [CrossRef]

10. Obata, S.; Tanaka, H.; Saiki, K. Electrical and spectroscopic investigations on the reduction mechanism of graphene oxide. Carbon 2013, 55, 126-132. [CrossRef]

11. Sankeshwar, A. Electronic thermal conductivity and thermopower of armchair graphene nanoribbons. Carbon 2013, 52, 201-208.

12. Tang, G.P.; Zhou, J.C.; Zhang, Z.H.; Deng, X.Q.; Fan, Z.Q. A theoretical investigation on the possible improvement of spin-filter effects by an electric field for a zigzag graphene nanoribbon with a line defect. Carbon 2013, 60, 94-101. [CrossRef]

13. Joh, H.I.; Lee, S.; Kim, T.W.; Hwang, S.Y.; Hahn, J.R. Synthesis and properties of an atomically thin carbon nanosheet similar to graphene and its promising use as an organic thin film transistor. Carbon 2013, 55, 299-304. [CrossRef]

14. Qureshi, A.; Kang, W.P.; Davidson, J.L.; Gurbuz, Y. Review on carbon-derived, solid-state, micro and nano sensors for electrochemical sensing applications. Diam. Relat. Mater. 2009, 18, 1401-1420. [CrossRef]

15. Sorkin, V.; Yong, W.Z. Graphene-based pressure nano-sensors. J. Mol. Modeling 2011, 17, 2825-2830. [CrossRef] [PubMed]

16. Yao, J.; Shen, X.; Wang, B.; Liu, H.; Wang, G. In situ chemical synthesis of $\mathrm{SnO}_{2}$-graphene nanocomposite as anode materials for lithiumion batteries. Electrochem. Commun. 2009, 11, 1849-1852. [CrossRef]

17. Sun, X.; Zhuang, L.; Welsher, K.; Robinson, J.T.; Goodwin, A.; Zaric, S.; Dai, H. Nano-graphene oxide for cellular imaging and drug delivery. Nano Res. 2008, 1, 203-212. [CrossRef]

18. Cai, D.; Wang, S.; Lian, P.; Zhu, X.; Li, D.; Yang, W.; Wang, H. Superhigh capacity and rate capability of high-level nitrogen-doped graphene sheets as anode materials for lithium-ion batteries. Electrochim. Acta 2013, 90, 492-497. [CrossRef]

19. Choi, H.J.; Jung, S.M.; Seo, J.M.; Chang, D.W.; Dai, L.; Baek, J.B. Graphene for energy conversion and storage in fuel cells and supercapacitors. Nano Energy 2012, 1, 534-551. [CrossRef]

20. Hong, B.H. Large-scale pattern growth of graphene films for stretchable transparent electrodes. Nature 2009, 457, 706-710.

21. Hu, J.M.; Yang, T.; Wang, J.; Huang, H.; Nan, C.W. Purely electric-field-driven perpendicular magnetization reversal. Nano Lett. 2015, 15, 616-622. [CrossRef] [PubMed]

22. Peng, Z.; Ma, L.; Fan, F.; Zhi, Z.; Cheng, P.; Loya, P.E.; Zheng, L.; Gong, Y.; Zhang, J.; Zhang, X. Fracture toughness of graphene. Nat. Commun. 2014, 5, 3782.

23. Rajasekaran, G.; Narayanan, P.; Parashar, A. Effect of point and line defects on mechanical and thermal properties of graphene: A review. Crit. Rev. Solid State Mater. Sci. 2016, 4, 47-71. [CrossRef]

24. Gass, M.H.; Bangert, U.; Bleloch, A.L.; Wang, P.; Nair, R.R.; Geim, A.K. Free-standing graphene at atomic resolution. Nat. Nanotechnol. 2008, 3, 676-681. [CrossRef] [PubMed]

25. Yazyev, O.V.; Louie, S.G. Topological defects in graphene: Dislocations and grain boundaries. Phys. Rev. B Condens. Matter 2010, 81, 195420. [CrossRef]

26. Lehtinen, P.O.; Foster, A.S.; Ayuela, A.; Krasheninnikov, A.; Nordlund, K.; Nieminen, R.M. Magnetic properties and diffusion of adatoms on a graphene sheet. Phys. Rev. Lett. 2003, 91, 17202. [CrossRef]

27. Yadav, S.; Zhu, Z.; Singh, C.V. Defect engineering of graphene for effective hydrogen storage. Int. J. Hydrogen Energy 2014, 39, 4981-4995. [CrossRef] 
28. Wang, M.C.; Yan, C.; Ma, L.; Hu, N.; Chen, M.W. Effect of defects on fracture strength of graphene sheets. Comput. Mater. Sci. 2012, 54, 236-239. [CrossRef]

29. Rahmandoust, M. Computational evaluation of the mechanical properties of synthesized graphene quantum dots under consideration of defects. Carbon Lett. 2021, 31, 427-440. [CrossRef]

30. Park, Y.; Hyun, S. Size effect of defects on the mechanical properties of graphene. J. Korean Phys. Soc. 2018, 72, 681-686. [CrossRef]

31. Motamedi, M.; Esfandiarpour, A. Effect of center crack on mechanical properties of graphene. J. Nano Res. 2018, 55, 22-31. [CrossRef]

32. Lopez-Polin, G.; Gomez-Navarro, C.; Parente, V.; Guinea, F.; Gomez-Herrero, J. Increasing the elastic modulus of graphene by controlled defect creation. Nat. Phys. 2015, 11, 26-31. [CrossRef]

33. Ng, T.Y.; Yeo, J.J.; Liu, Z.S. A molecular dynamics study of the thermal conductivity of graphene nanoribbons containing dispersed Stone-Thrower-Wales defects. Carbon 2012, 50, 4887-4893. [CrossRef]

34. Teng, Y.N.; Yeo, J.; Liu, Z. Molecular dynamics simulation of the thermal conductivity of shorts strips of graphene and silicene: A comparative study. Int. J. Mech. Mater. Des. 2013, 9, 105-114.

35. Lee, C.; Wei, X.; Kysar, J.W.; Hone, J. Measurement of the elastic properties and intrinsic strength of monolayer graphene. Science 2008, 321, 385-388. [CrossRef]

36. Georgakaki, D.; Ziogos, O.G.; Polatoglou, H.M. Vibrational and mechanical properties of Si/Ge nanowires as resonators: A molecular dynamics study. Phys. Status Solidi 2014, 211, 267-276. [CrossRef]

37. Zhan, H.F.; Gu, Y.T. A fundamental numerical and theoretical study for the vibrational properties of nanowires. J. Appl. Phys. 2012, 111, 124303. [CrossRef]

38. Sun, H.; Jin, Z.; Yang, C.; Akkermans, R.L.; Robertson, S.H.; Spenley, N.A.; Miller, S.; Todd, S.M. COMPASS II: Extended coverage for polymer and drug-like molecule databases. J. Mol. Modeling 2016, 22, 47. [CrossRef]

39. Pykal, M.; Jureka, P.; Karlick, F.; Otyepka, M. Modelling of graphene functionalization. Phys. Chem. Chem. Phys. 2015, 18, 6351-6372. [CrossRef] 